\title{
DETECTOR FOR WATER VAPOR IN CLOSED PIPES.
}

\author{
By E. R. Weaver and P. G. Ledig.
}

\section{ABSTRACT.}

A new instrument for indicating the amount of water vapor in a gas is described. The device is simple and especially suitable for use in high-pressure piping and other situations in which the determination of water vapor is usually attended with difficulty. It also serves to detect smaller amounts of water vapor than do most of the methods in common use.

The following paper describes a simple device for detecting the presence of water vapor, especially in such locations as the interior of pipes containing compressed gases.

The device was suggested by a description of the invention of Todd and Bousfield ${ }^{1}$ for the same purpose. The apparatus described in the patent consists of two gauze electrodes separated by a layer of granular calcium chloride or other hygroscopic salt inclosed in a tube through which the gas is passed. The contents of the tube remain nonconducting so long as the calcium chloride is dry, but in the presence of much water vapor the granules become coated with a continuous film of solution which establishes electrical connection between the electrodes and gives an indication of the moisture present. A somewhat different arrangement was used by Paul Anderson, ${ }^{2}$ who dipped the ends of two wires into the fused salt and exposed the bead of adhering salt to the gas to be tested. Two objections to the above arrangements, which it seemed possible to overcome, are (I) the time and the amount of water required to produce a definite effect, and (2) the difficulty of restoring the detector to its original condition for further testing. In addition, the device of Todd and Bousfield requires a definite circulation of gas through the tube.

The above objections are largely eliminated in the present apparatus, represented in Figure I, which is made of a piece of straight glass tubing sealed at one end. Near the closed end two platinum wires are sealed through the glass and fused against the outer surface of the tube, which is then frosted with a paste

1 Todd and Bousfield, Brit. Pat. 137547 of 1920.

2 Anderson. J. Chem. Soc., 121, pp. II53-Ir6r; r922. $59273^{\circ}-23$ 
of barium sulphate and ammonium fluoride. The ends of the platinum wires inside the tube are soldered or fused to copper leads. The outside of the cell is carefully platinized by applying a colloidal solution of platinic chloride in lavender oil, as described by McKelvy and Taylor. ${ }^{3}$ This process is repeated until the layer of platinum is entirely smooth and continuous, care being taken that the platinized surface is in good contact with the electrode wires. The tube is then coated with paraffin and a line etched around it between the two electrodes with hydrofluoric acid;

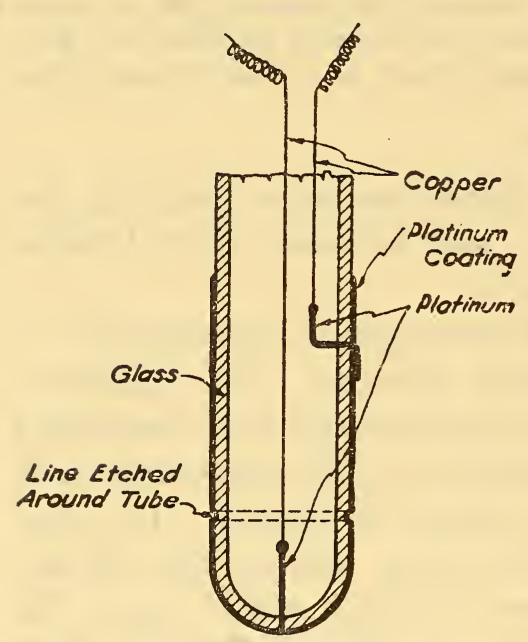

FIG. I.-Detector for water vapor.

The size of the tube is of little consequence. Those employed in the laboratory have usually been made from tubes of any convenient length, having about $8 \mathrm{~mm}$ outside and $5 \mathrm{~mm}$ inside diameter. current of the film bridging the "scratch" between the electrodes is determined in any suitable way and is a measure of the concentration of water vapor present.

For the purpose of studying the device in the laboratory, such a detector was used in the manner shown in Figure 2. The detector was mounted in a tube of slightly larger diameter, through which the gas to be tested could be streamed, and set up either in a constant-temperature bath or in the open air of the room. The electrical circuits are shown diagrammaticaliy in the figure. The detector formed one arm of a Wheatstone bridge employing I5volt, 6o-cycle alternating current, and an alternating-current galvanometer in the measuring circuit. The alternating-current gal-

${ }^{3}$ McKelvy and Taylor. J. Am. Chem. Soc., 42, p. 1366; 1920. 
vanometer used, when connected to a source of IIo-volt alternating current, supplies $\mathrm{I}_{5}$ volts (or lower potentials) directly from a "drop coil."

The detector was exposed to atmospheres of known moisture content by bubbling air through sulphuric-acid solutions of various concentrations. The solutions were made by diluting ordinary C. P. sulphuric acid with distilled water, were cooled to $20^{\circ} \mathrm{C}$., their density determined by the use of hydrometers, giving readings accurate to the third decimal place, and the percentage of

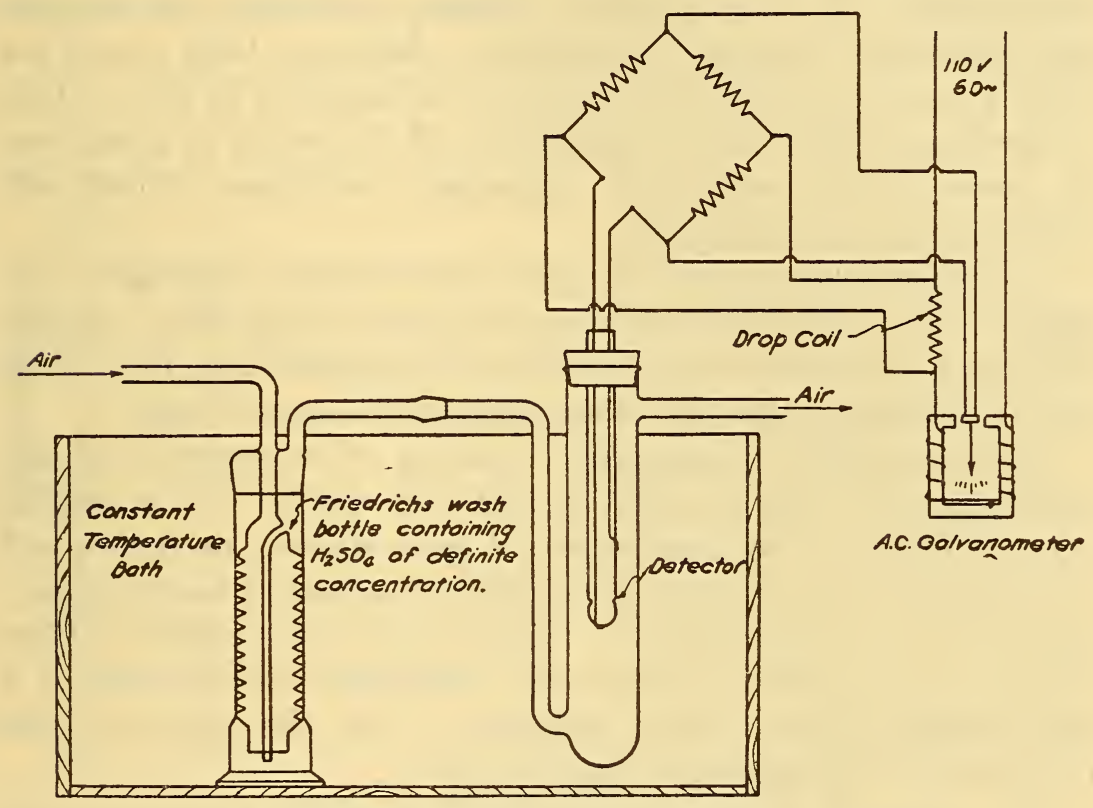

FIG. 2.-Simple arrangement of apparatus for laboratory study.

acid taken from the density table in Bureau of Standards Circular No. I9.

The water-vapor pressures of the solutions were obtained from the data of Regnault. ${ }^{4}$ The air was saturated to the watervapor pressure of the solutions by bubbling it through them in a Greiner-Friedrich spiral wash bottle; it was then passed directly to the tube containing the detector.

The first work with a detector of this form was done in I9rg. Two hygroscopic substances were used-phosphoric acid and rubidium carbonate-the latter being employed because it would not be greatly affected by traces of either carbon dioxide or

\footnotetext{
4 Regnault. Ann. Chim. Phys. (3), 15, p. I 79; r845; via Landolt Börnstein Tabellen.
} 
ammonia. It was at first hoped that the method could be made accurately quantitative. When it became certain that this could not be done the work was abandoned, but one of the detectors, with a coating of phosphoric acid, was left in place in the tube in which the test was made and stored in the laboratory for a period of three years. The gas inlet and outlet were open to the air during that time. Because of recent inquiries from different sources for a means of detecting moisture, particularly in gases compressed for liquefaction, it seemed worth while to renew the investigation of this detector. Without renewing the original phosphoric-acid coating, the detector, which had been stored for three years, was tried and found to be as sensitive as ever. This is excellent evidence for the reliability of the device as a detector of unusual and undesirable operating conditions, which will probably be its principal use.

In addition to phosphoric acid and rubidium carbonate, sulphuric acid, a mixture of sulphuric and phosphoric acids, calcium cholride and sodium and potassium hydroxides have been tried as hygroscopic electrolytes, using the same detector tubes.

When renewing or changing a coating of electrolyte, the detector tube was first carefully washed and dried. A thin gelatin solution of about the consistency of good ink was prepared, and to it enough of the hygroscopic electrolyte added to make approximately a I per cent solution. This solution was applied to the surface of separation between the electrodes with the tip of a camel's-hair brush barely moistened with the solution, and quickly dried by passing dry air over it.

The attempts to use the alkaline hydroxides as hygroscopic coatings were not a success, because the resistances of the films never approached equilibrium but continually increased. This is believed to have been caused by a small amount of carbon dioxide (or possibly other acid vapors from the sulphuric acid) which remained in the air stream in spite of some effort to eliminate it.

With any one electrolyte the resistance of the detector can, of course, be varied over a wide range by changing the strength and amount of the solution applied. The relative resistances, when in equilibrium with atmospheres of different humidities, are reasonably independent of the amount of electrolyte in the film and dependent only upon the hygroscopic substance used. How closely this relation applies is indicated by Figure 3, which shows the observed resistances of two coatings of sulphuric acid. One 
had 13.5 times the resistance of the other. The upper curve is drawn through the points representing the observed resistances of the thinner coating. The lower curve is then drawn I.3 divisions on the $\log$ scale $\left(\log \mathrm{I}_{3} .5=\mathrm{r} .3\right)$ below the upper one. The circles near the lower curve represent observed data on the thicker coating and indicate the close agreement with the rule stated.

Figure 4 shows the resistance curves for films of sulphuric acid, phosphoric acid, and a mixture of sulphuric and phosphoric acids containing about equal weights of each. This mixture was pre-

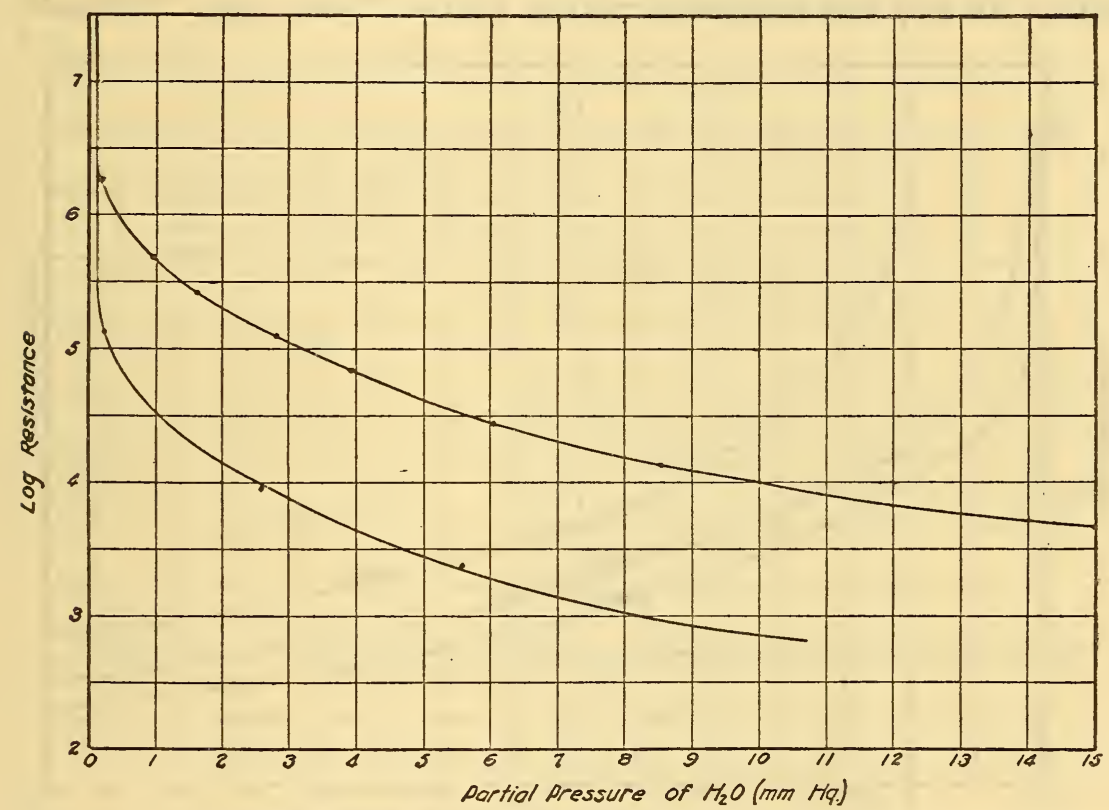

FIG. 3.-Resistances of sulphuric acid films in equilibrium with atmospheres of different humidities at $22^{\circ} \mathrm{C}$.

pared in the hope of extending the range within which a given film could be used for semiquantitative work. The rate at which changes of resistance take place when the humidity of the atmosphere is changed depend greatly upon the thickness of the hygroscopic film. The behavior in this respect of the 3 -year-old phosphoric-acid coating is shown in Figure 5. The readings were made by two observers, one of whom observed resistances while the other noted the stop watch and recorded readings. In the middle range of resistances about two hours was required to so nearly attain equilibrium that no further change of resistance could be noted by the rather sensitive method of measurement employed, but the change which took place after the first five 
minutes had little practical significance. On changing from an atmosphere containing about 0.9 per cent of water vapor to a dry atmosphere, the resistance increased about roo-fold in the first minute. On changing back to the former atmosphere, the resistance decreased to about one seven-hundredth of its maximum value in one minute. The resistance changes of the sulphuric-acid film represented in Figure 4 were even more rapid.

Probably the greatest drawback to the extensive use of the detector for quantitative work is the narrow range of high sensitivity for any one substance-0.I-5 $\mathrm{mm}$ of water-vapor pressure

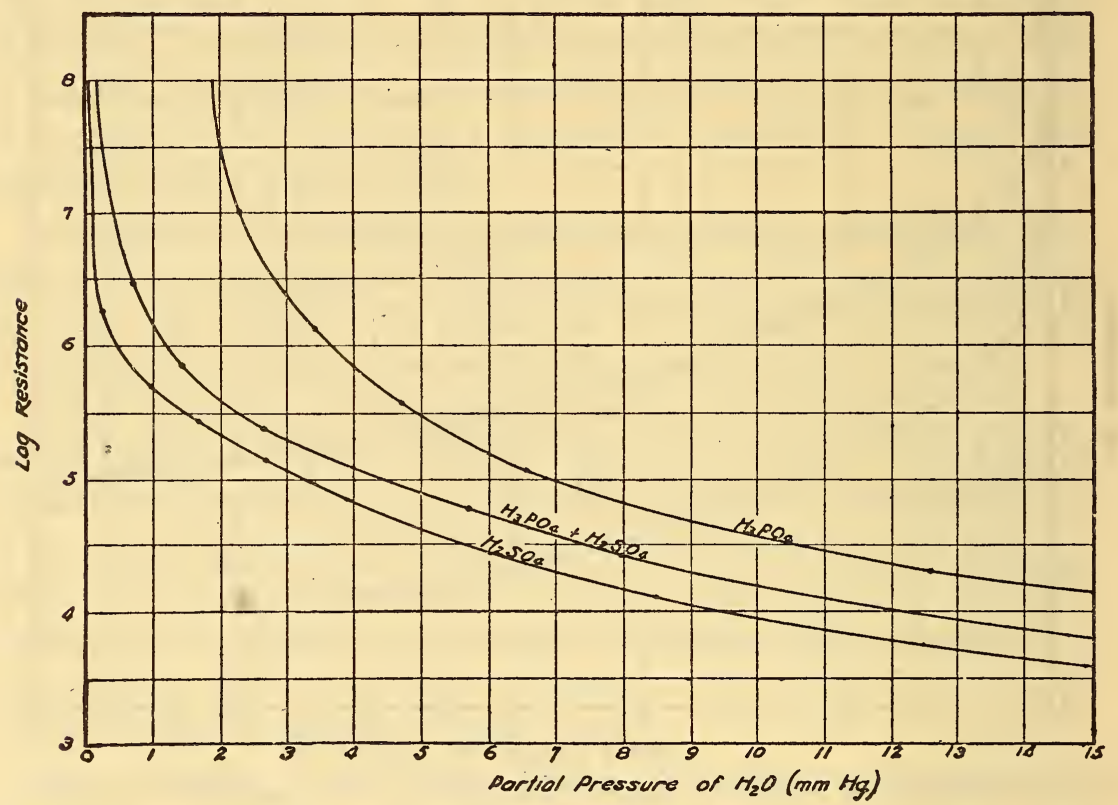

FIG. 4.-Resistances of films of sulphuric acid, phosphoric acid, and mixed sulphuric and phosphoric acids at $22^{\circ} \mathrm{C}$.

for sulphuric acid, 2-Io $\mathrm{mm}$ for phosporic acid, and even narrower ranges for salts, like rubidium carbonate and calcium chloride. The sensitive range of rubidium carbonate is slightly higher than that of phosphoric acid (it was not very accurately determined); that of calcium chloride is in the neighborhood of $15 \mathrm{~mm}$.

It was found to be impossible to let a film come to equilibrium with an atmosphere containing much water and to subsequently reproduce the resistance readings earlier obtained at low humidities. In every case, the resistance increased under such conditions. It appears probable that the electrolyte flows off the surface of the cell when it is allowed to absorb too much water; 
but if the coating is kept dry, or not far from its sensitive range resistance readings are quite uniform and reproducible.

Another important disadvantage of the detector is the large effect of temperature. In the case of phosphoric acid and the hygroscopic salts, an increase of temperature expels water from the film and greatly increases its resistance. With sulphuric-acid films, the effect of temperature upon the composition of the film and the resistivity of a solution of given composition are more nearly balanced and the resultant coefficient is not nearly so great.

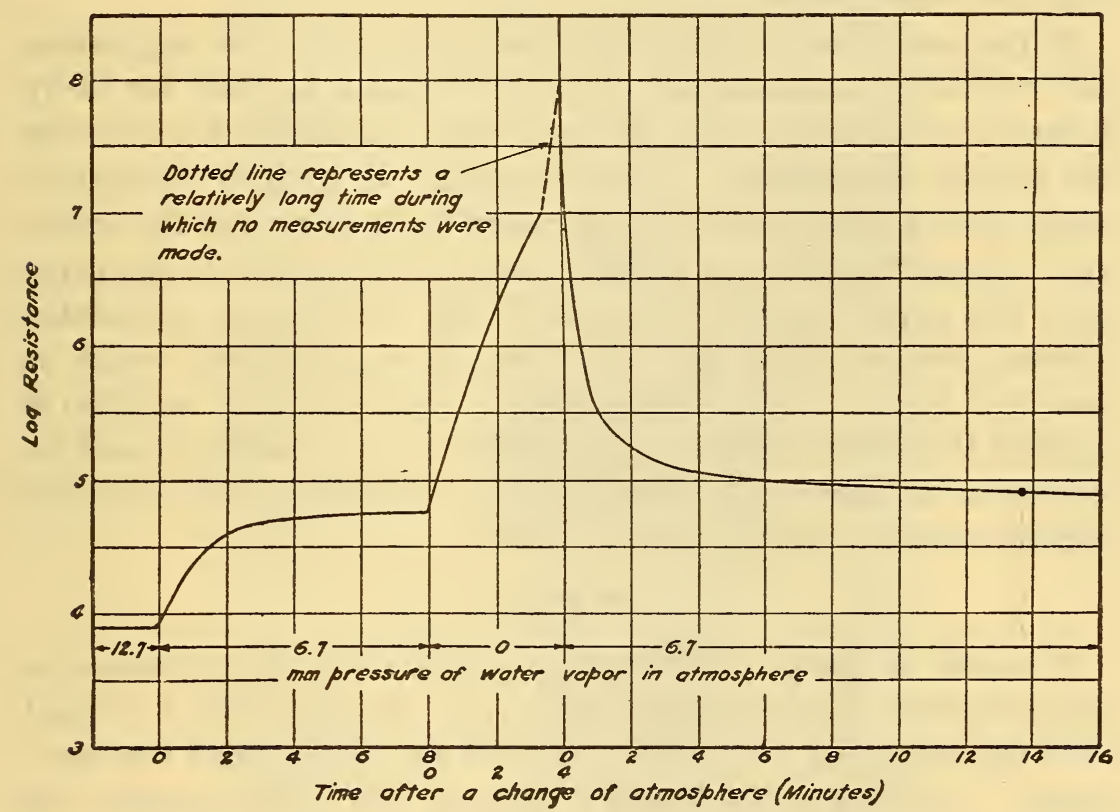

FIG. 5.-Rate of change of resistance following a sudden change of atmosphere.

The curves for sulphuric and phosphoric acids in Figure 4 can be used in connection with a single determination of resistance at known humidity to establish a calibration curve for any detector using the same compounds at about the same temperature, $22^{\circ} \mathrm{C}$. If, for example, a detector with a sulphuric-acid film which showed a resistance $\mathrm{R}$ in an atmosphere known to have a water-vapor pressure of $5 \mathrm{~mm}$ shows a resistance of ro $\mathrm{R}$ when placed in a pipe line, it can be at once seen from the curve that the pressure of the water vapor in the pipe must be about I.I mm. A resistance of $100 \mathrm{R}$ would correspond to a vapor pressure of o.I $\mathrm{mm}$.

In case such a detector is used regularly, it should be checked with reasonable frequency by exposing it to an atmosphere of 
known humidity. It is especially necessary to make this check after the detector is known to have been in an atmosphere of unusually high humidity. The coating should be replaced whenever there is reason to suspect that appreciable chemical change has taken place or when the resistance is no longer in the most easily measurable range.

Figure 6 is a photograph of a detector prepared for insertion into a pipe line at high pressure. The glass tube is platinized and soldered into the steel bushing by the method described by McKelvy and Taylor. ${ }^{5}$

If the same device were combined with means for regulating and measuring temperature, it could certainly be used for fairly accurate quantitative work at any range of humidity by selecting the proper electrolyte. If, for example, an entirely nonhygroscopic salt of slight solubility were used for coating and the resistance always brought to a definite value by changing the temperature, the apparatus would be practically a dew-point apparatus of small size on which the first trace of condensation would be detected by a much more sensitive and generally applicable method than direct vision. In this form, the apparatus could be located at a place inaccessible for visual observation and its indications recorded electrically if desired.

\section{SUMMARY.}

A simple device is described for determining the approximate concentration of water vapor in a gas. A glass tube is coated with platinum and the coating divided by etching into two electrodes. Platinum wires sealed through the glass connect the electrodes to a measuring circuit. The resistance to alternating current of a thin film of a hygroscopic electrolyte bridging the gap between the electrodes is used as the measure of the water vapor in the atmosphere with which the film is in contact. Sulphuric and phosphoric acids and various hygroscopic salts can be used in forming the conducting film. The detector is simple, rugged, and easily adapted for use in high-pressure piping and other situations in which the determination of water vapor is usually attended with difficulty.

Laboratory experiments showing the reliability, method of application, and limitations of the device are described.

WASHINGTON, February 24, 1923.

\footnotetext{
${ }^{6} \mathrm{~J}$. Am. Chem. Soc., 42, p. I366; 1920.
} 
Technologic Papers of the Bureau of Standards, Vol. 17.

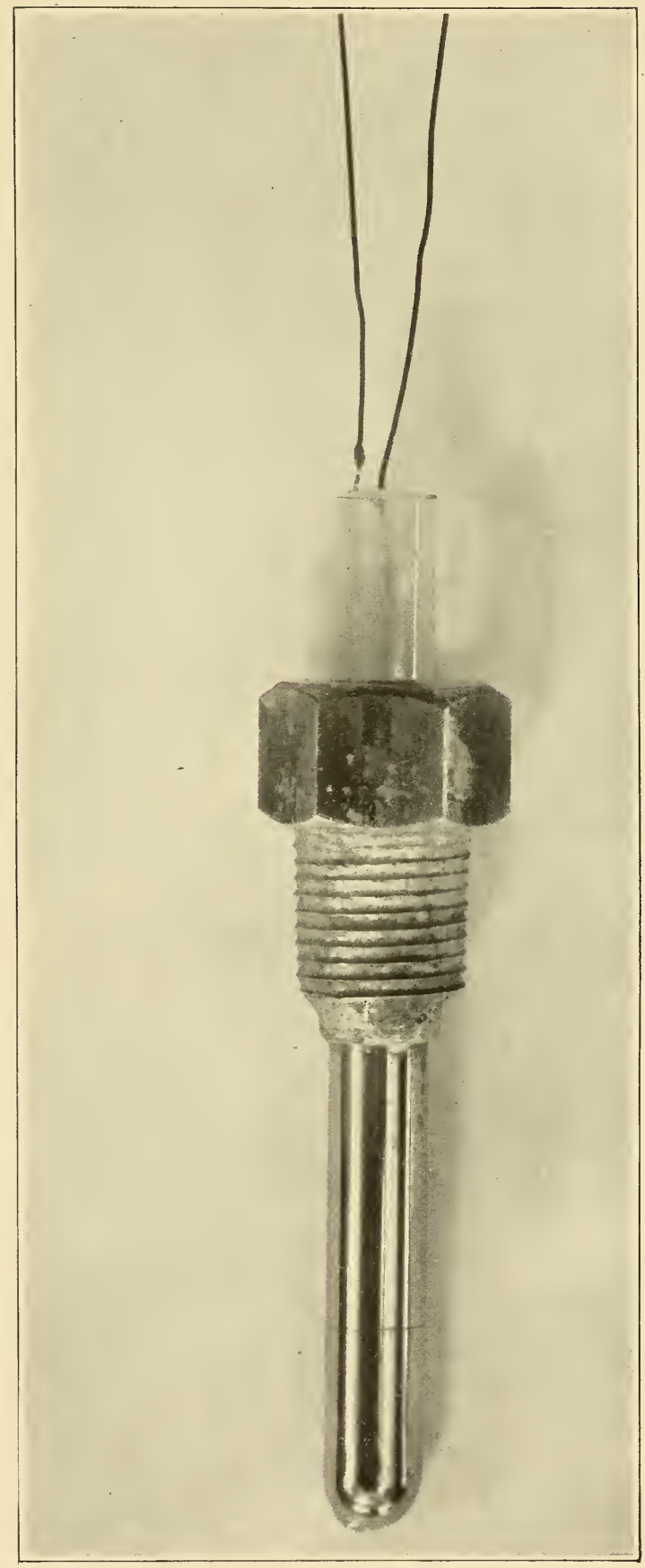

FIG. 6.-Dctector in bushing ready for insertion into high-pressure piping. 